\title{
The Prevalence of Hypertension in Endodontic Clinics: A Pilot Study
}

\author{
Dania F Bogari ${ }^{1}$, Ghaida T Bakalka ${ }^{2}$, Loai W Hazzazi ${ }^{3}$, Ahmed M Jan ${ }^{4}$, Wael Y Elias ${ }^{5}$, Neville J McDonald ${ }^{6}$ and Turki Y Alhazzazi $^{3^{*}}$ \\ ${ }^{1}$ Department of Endodontics, King Abdulaziz University, Faculty of Dentistry, Jeddah, Saudi Arabia \\ ${ }^{2}$ King Abdulaziz Medical City, National Guard Hospital, Jeddah, Saudi Arabia \\ ${ }^{3}$ Department of Oral Biology, King Abdulaziz University, Faculty of Dentistry, Jeddah, Saudi Arabia \\ ${ }^{4}$ Department of Oral and Maxillofacial Surgery, King Abdulaziz University, Faculty of Dentistry, Jeddah, Saudi Arabia \\ ${ }^{5}$ Department of Diagnostic Oral Sciences, King Abdulaziz University, Faculty of Dentistry, Jeddah, Saudi Arabia \\ ${ }^{6}$ Cariology, Restorative Sciences, and Endodontics Department, University of Michigan, Ann Arbor, Michigan 48109, USA
}

"Corresponding author: Turki Y Alhazzazi, BDS, FRCD(C), PhD, Assistant Professor and Consultant in Endodontics, Chairman, Oral Biology Department, Faculty of Dentistry, King Abdulaziz University, Jeddah, Saudi Arabia, Tel: 00966-2-6403443 (22024), 0096 6505649093; E-mail: talhazzazi@kau.edu.sa

Rec date: Mar 21, 2016; Acc date: Apr 07, 2016; Pub date: Apr 13, 2016

Copyright: ( 2016 Bogari DF, et al. This is an open-access article distributed under the terms of the Creative Commons Attribution License, which permits unrestricted use, distribution, and reproduction in any medium, provided the original author and source are credited.

\begin{abstract}
Background: Hypertension is one of the leading causes of death worldwide, and a major risk factor for heart diseases and strokes. A lot of undiagnosed or uncontrolled hypertensive patients seek dental treatment in regular basis. However, neither doctors nor patients are doing enough to fight and diagnose this silent killer disease. This study investigated the prevalence of undiagnosed or uncontrolled hypertensive patients who visited our endodontic clinic for dental treatment.
\end{abstract}

Methods: 102 patients were included in this study. Patients were non-smokers and only presented a history of hypertension alone or a clear medical history. We measured blood pressure using a digital sphygmomanometer after patients had been seated in a dental chair for at least 10 minutes. We present the data as descriptive and percentage values.

Results: A large fraction of patients $(63.7 \%)$ were found to be hypertensive irrespective of their awareness of having hypertension. Nearly half $(52.9 \%)$ of participants were unaware they had hypertension, and only $10.8 \%$ of patients knew they had hypertension. Only approximately half of the latter group of patients $(4.9 \%)$ was already receiving medication for their hypertension.

Conclusion: Our data reveals that dentists play a critical role in detecting undiagnosed patients with hypertension; this role should be emphasized in our specialty as a standard of care.

Keywords: High blood pressure; Hypertension; Endodontics; Dentistry; Root canal treatment

\section{Introduction}

High blood pressure or hypertension is the second leading cause of death in the United States after tobacco, and it is a major risk factor for heart disease and stroke [1]. It is estimated that 1 out of every 3 persons is diagnosed with high blood pressure in the United States and worldwide. High blood pressure is a prime predisposing factor of $45 \%$ of deaths due to cardiovascular disease and $~ 51 \%$ of deaths due to stroke. In addition, fewer than $50 \%$ of patients who are aware of their elevated blood pressure are currently taking efforts to control it [2]. However, neither doctors nor patients are doing enough to fight and diagnose this disease, which represents a silent killer. As a result, increasing awareness and knowledge about the predisposing factors of this silent killer are essential to prevent heath complications and death.

The role of dentists in diagnosing and assessing risk factors for important and life-threatening medical conditions such as head and neck cancer and hypertension is essential and should be considered to be a part of standard care. Unfortunately, this situation is not always the case. Only a few dentists are actually aware of the importance of this public health disease.

Many patients with undiagnosed or uncontrolled high blood pressure seek dental treatment on a regular basis. Individuals with high blood pressure are at risk of developing medical complications such as heart attack, stroke, or renal disease during or after dental treatment, and their medical status may affect the dental treatment outcomes. A study conducted in Nigeria reported hypertension in approximately $20 \%$ of dental patients; half of these cases were undiagnosed until they were examined at the dental clinic [3]. Similarly, in another study, approximately $29 \%$ of patients were reported to have hypertension when examined in a dental clinic; $73 \%$ of these patients were referred to a family physician because they were unaware of their medical condition [4]. Therefore, it seems that dentists may play a crucial role in diagnosing this silent killer disease. The goal of our study was accordingly to investigate the prevalence of hypertensive patients who sought endodontic treatment at Almashfa Dental Clinic, and identify the percentage of individuals who were unaware of their high blood pressure before undergoing root canal treatment at Almashfa Dental Clinic. 
Page 2 of 4

\section{Materials and Methods}

\section{Sampling size and inclusion criteria}

This retrospective study was conducted using data obtained from the records of all patients who visited the Almashfa Dental Clinic in Jeddah, Saudi Arabia seeking endodontic treatment from March 1st, 2015 to May 31st, 2015 ( $\mathrm{n}=102)$. The study included male and female patients who reported a clear medical history or a history of hypertension. None of the patients included in the study were smokers. We did not impose any restrictions on the ages of patients for reasons that will be noted in the later in the article.

\section{Protocol}

We obtained written consent from all of the patients that their information could be used in this study, and the procedures that we conducted were accordingly part of our daily routine work. This study was approved by the Executive Board Ethical Committee Members of Almashfa Dental Clinic and was in full accordance with the World Medical Association Declaration of Helsinki.

After recording the patient's chief complaints and medical and dental history, we obtained a blood pressure measurement using a digital sphygmomanometer. Patients were seated comfortably in an upright position, and their legs were uncrossed. Readings were obtained after the patients had been seated in the clinic for at least 10 minutes. If the reading that we obtained was not within the normal range for either systolic/diastolic or both (Table 1), the reading was retaken 5 minutes later and the average was recorded [5]. The raw data were entered into Excel (Microsoft version 14.5.20) and were presented as descriptive and percentage values.

\begin{tabular}{|c|c|c|}
\hline Classifications & $\begin{array}{c}\text { Systolic blood pressure } \\
\mathbf{( m m ~ H g )}\end{array}$ & $\begin{array}{c}\text { Diastolic blood } \\
\text { pressure } \mathbf{( m m ~ H g})\end{array}$ \\
\hline Normal & $<120$ & $<80$ \\
\hline Pre-hypertension & $120-139$ & $80-89$ \\
\hline Stage 1 hypertension & $140-159$ & $90-99$ \\
\hline Stage 2 hypertension & $>160$ & $>100$ \\
\hline
\end{tabular}

Table 1: Hypertension classification.

\section{Results}

The hypertension classification that we employed was based on a study by Chobanian et al. as shown in Table 1 [5]. The characteristics and summary of our study samples $(\mathrm{n}=102)$ are presented in Table 2. The mean age of our study group was $40.9 \pm 6.7$ years, and $69 \%$ of the patients were female and $31 \%$ of the patients were male.

We found that a large cohort of patients were hypertensive $(63.7 \%$; Figure 1); $52.9 \%$ of these patients were unaware they were hypertensive, and $10.8 \%$ knew they were hypertensive. Only approximately half of the latter group of patients (4.9\%) were already on medication(s) to treat their hypertension; $5.9 \%$ of patients were not taking any hypertension medication. We found that the majority of patients were in the pre-hypertensive stage (35\%); $10 \%$ and $19 \%$ of patients were in hypertension stages 1 and 2, respectively (Figure 2). Data showing the differences between males and females in the hypertension sub categorization status are presented in Figure 3.

\begin{tabular}{|c|c|c|c|c|c|}
\hline Variables & $\mathbf{N}$ & Min & Max & Mean & SD \\
\hline Age & 99 & 13 & 85 & 40.96 & 16.7 \\
\hline $\begin{array}{l}\text { Systolic Blood } \\
\text { Pressure }\end{array}$ & 102 & 97 & 171 & 127.44 & 17.7 \\
\hline $\begin{array}{l}\text { Diastolic Blood } \\
\text { Pressure }\end{array}$ & 102 & 56 & 105 & 75.34 & 11.6 \\
\hline HR & 102 & 51 & 114 & 81.59 & 13.2 \\
\hline \multicolumn{2}{|l|}{ Total } & \multicolumn{2}{|l|}{102} & \multicolumn{2}{|l|}{$100 \%$} \\
\hline \multirow{4}{*}{$\begin{array}{l}\text { Systolic Blood } \\
\text { Pressure }\end{array}$} & Normal & \multicolumn{2}{|l|}{40} & \multicolumn{2}{|l|}{$39.20 \%$} \\
\hline & Pre-hypertension & \multicolumn{2}{|l|}{39} & \multicolumn{2}{|l|}{$38.20 \%$} \\
\hline & Stage 1 hypertension & \multicolumn{2}{|l|}{16} & \multicolumn{2}{|l|}{$15.70 \%$} \\
\hline & Stage 2 hypertension & \multicolumn{2}{|l|}{7} & \multicolumn{2}{|l|}{$6.90 \%$} \\
\hline \multirow{4}{*}{$\begin{array}{l}\text { Diastolic Blood } \\
\text { Pressure }\end{array}$} & Normal & \multicolumn{2}{|l|}{69} & \multicolumn{2}{|l|}{$67.60 \%$} \\
\hline & Pre-hypertension & \multicolumn{2}{|l|}{18} & \multicolumn{2}{|l|}{$17.60 \%$} \\
\hline & Stage 1 hypertension & \multicolumn{2}{|l|}{10} & \multicolumn{2}{|l|}{$9.80 \%$} \\
\hline & Stage 2 hypertension & \multicolumn{2}{|l|}{5} & \multicolumn{2}{|l|}{$4.90 \%$} \\
\hline \multirow{4}{*}{ Overall Status $1^{\$}$} & Normal & \multicolumn{2}{|l|}{37} & \multicolumn{2}{|l|}{$36.30 \%$} \\
\hline & Pre-hypertension & \multicolumn{2}{|l|}{36} & \multicolumn{2}{|l|}{$35.30 \%$} \\
\hline & Stage 1 hypertension & \multicolumn{2}{|l|}{18} & \multicolumn{2}{|l|}{$17.60 \%$} \\
\hline & Stage 2 hypertension & \multicolumn{2}{|l|}{11} & \multicolumn{2}{|l|}{$10.80 \%$} \\
\hline \multirow{2}{*}{ Overall Status $2^{\#}$} & Normal & \multicolumn{2}{|l|}{37} & \multicolumn{2}{|l|}{$36.30 \%$} \\
\hline & Hypertensive & \multicolumn{2}{|l|}{65} & \multicolumn{2}{|l|}{$63.70 \%$} \\
\hline \multicolumn{6}{|c|}{$\begin{array}{l}\text { \$Overall subcategorisation of diagnosed hypertension in both systolic and } \\
\text { diastolic cases (combined) }\end{array}$} \\
\hline
\end{tabular}

Table 2: Characteristics and data summary of the 102 patients.

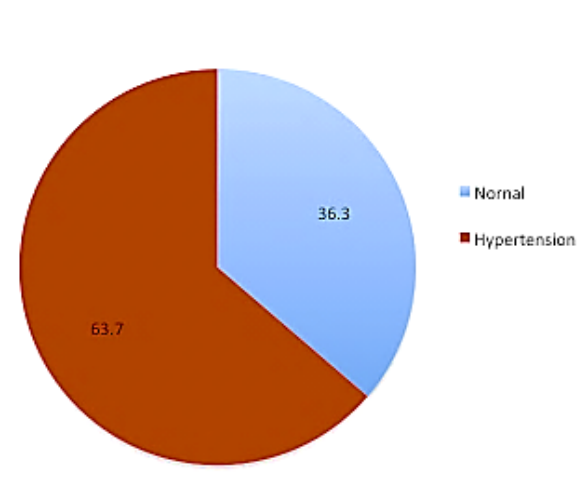

Figure 1: Overall hypertension of the examined group (\%). 


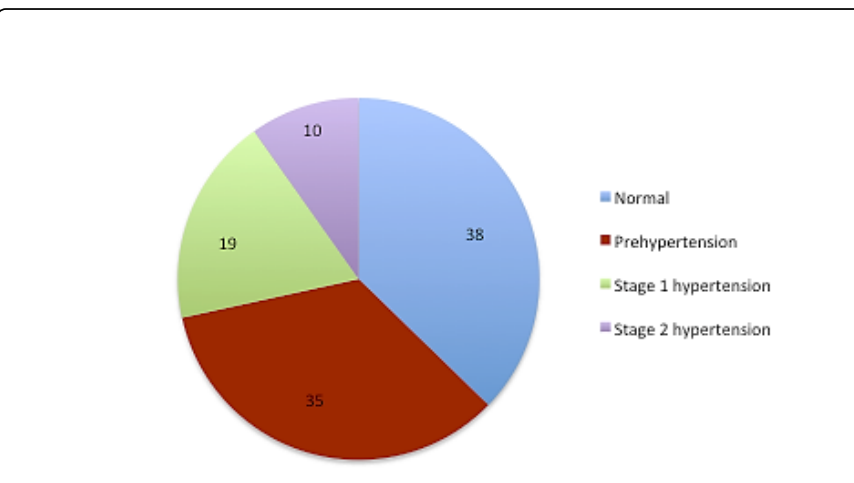

Figure 2: Sub categorization of hypertension status of the examined group (\%).

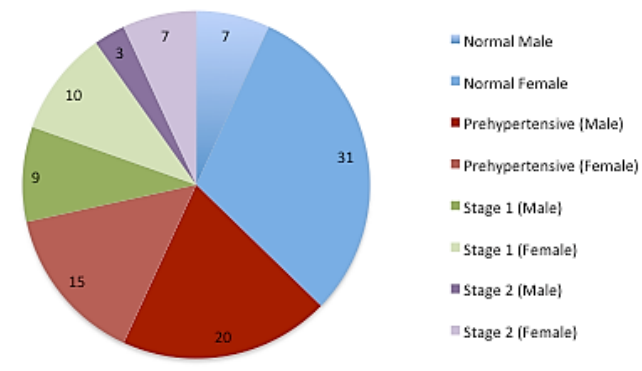

Figure 3: The differences between males and females in the hypertension sub categorization status (\%).

\section{Discussion}

Few studies have reported the prevalence of hypertensive patients seeking dental treatment in general. To the best of our knowledge, our study is the first to report the prevalence of hypertension patients in an endodontic clinic. Our results revealed that the prevalence of elevated blood pressure readings among screened patients in our endodontic clinic was $63.7 \%$. Nearly $52.9 \%$ of these patients were unaware they had hypertension. Similar results have been reported at the University of Michigan in a study conducted at the School of Dentistry in the Adult Comprehensive Care Clinics. 32\% of the studied sample was recorded as being hypertensive, and $49 \%$ of these patients were unaware of their high blood pressure prior to being screened at the dental clinic [6]. In a study conducted in Spain, approximately $29 \%$ of the studied patients were revealed to have hypertension when examined in a dental clinic. However, $73 \%$ of these patients were referred to a family physician because they were unaware of their medical condition [4]. Similarly, in a study conducted in Nigeria, approximately $52.5 \%$ of patients were unaware of their hypertensive status, although the overall prevalence of hypertension (19.7\%) was lower than that of other studies [3]. In addition, a study conducted at King Abdulaziz University, Faculty of Dentistry, Jeddah, reported that $37 \%$ of their screened female patients were pre-hypertensive and that
$18 \%$ of patients had high blood pressure $(\geq 140 / 90 \mathrm{~mm} \mathrm{Hg})$; of those patients, $66 \%$ were unaware of their medical condition [7].

One of the best studies to raise awareness of hypertension among general dentists was conducted by Michael et al. [8]. They studied 1,343 patients from 16 dental offices over a 2 month period. They found hypertension mostly among male patients aged 50-59 years and female patients aged 40-69 years. Approximately $12 \%$ of patients had high blood pressure. Of these patients, 35\% were diagnosed with hypertension as a result of the screening process and confirmed to be unaware of their medical condition prior to the dental screening [8]. Although there are differences in the prevalence of hypertension among reported studies, the percentage of undiagnosed hypertension remains high and averages about $50 \%$. Differences in this prevalence may be due to variations in sample size and the knowledge, awareness, and socioeconomic status of patients. Additionally, it is known that the prevalence of hypertension is lower in high-income countries [2]. Although all patients in our study were considered to be of high socioeconomic status, we still recovered a high percentage of hypertension in our cohort. Luckily, a high percentage of our patients were only in the pre-hypertensive stage (35.3\%). However, strikingly, several of the diagnosed patients who were unaware of their condition and were in hypertension stage 1 or 2 were medical physicians or spouses of medical physicians. These individuals are now receiving hypertension treatment. Some people may argue that this increase in blood pressure maybe transient and not real, especially in the prehypertensive group. The argument is that this increase is due to anxiety of dental treatment or white coat hypertension phenomenon. We are aware of that fact; there is a prevalence of white coat hypertension of approximately $15 \%$ depending on age $[9,10]$.

Even in children, the prevalence of white coat hypertension has been reported to reach $47 \%$, and there is a strong association between hypertension and obesity [11-14]. However, irrespective of whether this increase in blood pressure is transient or not, this subject should still be taken seriously because it could increase the risk of cardiovascular events during dental treatment. In normotensive individuals, the intimal/medial wall thickness is $0.76 \mathrm{~mm}$; in patients with white coat hypertension, the intimal/medial wall thickness is 0.84 $\mathrm{mm}$, however, in hypertensive patients, the intimal/medial wall thickness is $0.98 \mathrm{~mm}$ [15]. This finding confirms that even a transient increase in blood pressure can cause harmful effects on the heart and increase the risk of unfavorable events during dental treatment. In addition, several studies support that white coat hypertension may be representative of an alarming sign of permanent hypertensive stage $[16,17]$. Therefore, measuring blood pressure in dental settings is highly recommended and should be part of a routine dental examination to reduce the number of cases that go undiagnosed. Furthermore, dentists should be aware of how to manage these patients, especially the ones with cardiovascular diseases, and consider revising the treatment protocol depending on patient's medication and disease status $[18,19]$. Hence, this will then help to decrease the number of incidents of serious life-threatening diseases and complications such cardiovascular diseases and strokes during or after dental treatment.

We advocate that dentists be more aware and knowledgeable about hypertension management and treatment in the dental office. Stress reduction protocols should be applied and considered for all patients to reduce the chances of complications, especially hypertensive ones, arising during or after dental treatment $[20,21]$. One of these measures may include the use of a low dose of midazolam to control anxiety 
[22]. Furthermore, hypertensive patients seem to possess a different background when it comes to oral health. It has been reported that the number of teeth lost by hypertensive male patients was significantly higher than zero, which was not the case when it came to female patients [23]. It appears that the prevalence of root canal treatments and apical periodontitis in smoker hypertensive patients is significantly higher than that in hypertensive non-smoker [24]. However, this association was not shown to be true when comparing non-smoker hypertensive patients and non-hypertensive normal controls [25]. In addition, the risk of tooth extraction after non-surgical root canal treatment was significantly associated with patients having medical conditions such as diabetes and hypertension [26]. This finding suggests that endodontists should be aware of patients' medical conditions prior to discussing anything in regard to treatment outcomes. Additionally, being aware of the evolving understanding of blood pressure targeted reading values is crucial before planning for dental treatment [19].

This pilot study is the evidence that hypertension screening needs to be taken more seriously by the dental profession. Our group is currently working on a large project that includes both governmental and dental sectors. This work will allow us to include more patients with different educational and socioeconomic backgrounds and to recruit more dentists with the aim of raising awareness about this silent killer disease.

\section{Conclusions}

Most of the public is aware of the importance of visiting a dentist once or twice a year for teeth cleanings and routine checkups to prevent dental caries. On the other hand, only a few individuals may visit their general physician for a routine checkup in the absence of any serious issues. In addition, only few people aware of the risk of hypertension may realize that they are walking around with this disease undiagnosed. Therefore, the role of dentists in detecting undiagnosed hypertension is crucial and should be emphasized in our specialty as a standard of care. Doing so will hopefully help to decreasing the risk of death from this silent killer disease worldwide and prevent the development of life-threatening complications such as strokes or cardiovascular diseases during or after dental treatment.

\section{Acknowledgments}

We would like to thank Kalvin Balucanag and his team for helping us with data tabulation and analysis. This research received no specific grant from any funding agency in the public, commercial, or not for profit sectors.

\section{References}

1. Institute of Medicine (2010) A population-based policy and systems change approach to prevent and control hypertension. Health and Medicine Division, National Academy of Sciences, Washington, DC.

2. World Health Organization (2013). A global brief on Hypertension. Silent killer, global public health crisis.

3. Ojehanon PI, Akhionbare O (2007) Hypertension among dental patients attending tertiary health institution in Edo State, Nigeria. Niger J Clin Pract 10: 220-223.

4. Fernández-Feijoo J, Núñez-Orjales J, Limeres-Posse J, Pérez-Serrano E, Tomás-Carmona I (2010) Screening for hypertension in a primary care dental clinic. Med Oral Patol Oral Cir Bucal 15: e467-e472.
5. Chobanian AV, Bakris GL, Black HR, Cushman WC, Green LA, et al. (2003) The seventh report of the Joint National Committee on prevention, detection, evaluation, and treatment of high blood pressure: the JNC 7 report. JAMA 289: 2560-2572.

6. Kellog SD, Gobetti JP (2004) Hypertension in a dental school patient population. J Dent Educ 68: 956-964.

7. Al-Zahrani MS (2011) Prehypertension and undiagnosed hypertension in a sample of dental school female patients. Int J Dent Hyg 9: 74-78.

8. Berman CL, Guarino M, Giovanelli S (1973) Hypertension detection by dentists. J N J Dent Assoc 44: 17.

9. Jurko A Jr, Minarik M, Jurko T, Tonhajzerova I (2016) White coat hypertension in pediatrics. Ital J Pediatr 42: 4.

10. Fisher M, Blackwell J, Saseen J (2005) Clinical inquiries. What is the best way to identify patients with white-coat hypertension? J Fam Pract 54: 549-550.

11. Matsuoka S, Kawamura K, Honda M, Awazu M (2002) White coat effect and white coat hypertension in pediatric patients. Pediatr Nephrol 17: 950-953.

12. Verma V, Sinah SK (2014) Influence of lean body-mass index versus that of fat mass index on blood pressure of gujarati school going adolescents. Indian J Physiol Pharmacol 58: 39-44.

13. Yamaguchi Y, Awazu M, Matsuoka S, Maeda J, Tokumura M, et al. (1999) White coat hypertension in two adolescents. Pediatr Nephrol 13: 60-62.

14. Schusterova I, Jurko A, Minarik M (2013) Left ventricular systolic and diastolic function in children with overweight and obesity. Bratisl Lek Listy 114: 526-530.

15. Chrysant SG (2006) White coat hypertension and white coat worse hypertension. In: Advanced therapy in hypertension and vascular disease. B.C. Decker Inc., Hamilton, Ontario.

16. Li L, Guo LZ, Li J, Wang Y, Liu, X, et al. (2015) White coat hypertension and obstructive sleep apnea. Sleep Breath 19: 1199-1203.

17. Martin CA, McGrath BP (2014) White-coat hypertension. Clin Exp Pharmacol Physiol 41: 22-29.

18. Munoz MM, Soriano YJ, Roda RP, Sarrión G (2008) Cardiovascular diseases in dental practice. Practical considerations. Med Oral Pathol Oral Cir Bucal 13: E296-E302.

19. Scicchitano P, Gesualdo M, Carbonara S, Palmiero P, Nazzaro P, et al. (2015) What's new and what gaps in 2013 European guidelines for the management of arterial hypertension: A reappraisal. Cardiology and Angiology: An International Journal 3: 181-191.

20. Little JW (2000) The impact on dentistry of recent advances in the management of hypertension. Oral Surg Oral Med Oral Pathol Oral Radiol Endod 90: 591-599.

21. Nichols C (1997) Dentistry and hypertension. J Am Dent Assoc 128: 1557-1562.

22. Watanabe $Y$, Higuchi H, Ishii-Maruhama M, Honda Y, Yabuki-Kawase A, et al. (2016) Effect of a low dose of midazolam on high blood pressure in dental patients: a randomised, double-blind, placebo-controlled, twocentre study. Br J Oral Maxillofac Surg.

23. Volzke H, Schwahn C, Dorr M, Schwarz S, Robinson D, et al. (2006) Gender differences in the relation between number of teeth and systolic blood pressure. J Hypertens 24: 1257-1263.

24. Segura-Egea JJ, Castellanos-Cosano L, Velasco-Ortega E, Rios-Santos JV, Llamas-Carreras JM, et al. (2011) Relationship between smoking and endodontic variables in hypertensive patients. J Endod 37: 764-777.

25. Segura-Egea JJ, Jimenez-Moreno E, Calvo-Monroy C, Rios-Santos JV, Velasco-Ortega E, et al. (2010) Hypertension and dental periapical condition. J Endod 36: 1800-1804.

26. Wang CH, Chueh LH, Chen SC, Feng YC, Hsiao CK, et al. (2011) Impact of diabetes mellitus, hypertension, and coronary artery disease on tooth extraction after nonsurgical endodontic treatment. J Endod 37: 1-5. 